\title{
Chemical Profiles of Incense Smoke Ingredients from Agarwood by Headspace Gas Chromatography-Tandem Mass Spectrometry
}

\author{
Wen-Yi Kao ${ }^{1,2,+}$, Chien-Yun Hsiang ${ }^{3,+}$, Shih-Ching Ho ${ }^{2}$, Tin-Yun Ho ${ }^{4, *}$ and Kung-Ta Lee ${ }^{1, *}$ \\ 1 Department of Biochemical Science and Technology, National Taiwan University, Taipei 10617, Taiwan; \\ mbkwi@dcb.org.tw \\ 2 Development Center for Biotechnology, New Taipei City 22180, Taiwan; scho@dcb.org.tw \\ 3 Department of Microbiology, China Medical University, Taichung 40402, Taiwan; cyhsiang@mail.cmu.edu.tw \\ 4 Graduate Institute of Chinese Medicine, China Medical University, Taichung 40402, Taiwan \\ * Correspondence: tyh@mail.cmu.edu.tw (T.-Y.H.); ktlee@ntu.edu.tw (K.-T.L.); \\ Tel.: +886-422-053-366 (ext. 3302) (T.-Y.H.); +886-233-664-436 (K.-T.L.) \\ $+\quad$ These authors contributed equally to this work.
}

Received: 26 October 2018; Accepted: 13 November 2018; Published: 14 November 2018

\begin{abstract}
Agarwood, the resinous wood in the heartwood of Aquilaria trees, has been used as incense in traditional Chinese medicine for its sedative, aphrodisiac, carminative, and anti-emetic effects. Grading of agarwood is usually based on its physical properties. Therefore, it is important to develop analytic methods for judgment and grading of agarwood. Here, we created a headspace (HS) preheating system that is combined with gas chromatography-mass spectrometry (HS GC-MS) to analyze the chemical constituents in the incense smoke produced by agarwood. Incense smoke generated in the HS preheating system was injected directly to GC-MS for analysis. A total of 40 compounds were identified in the incense smoke produced by Kynam agarwood, the best agarwood in the world. About half of the compounds are aromatics and sesquiterpenes. By analyzing chemical constituents in the incense smoke produced by Vietnamese, Lao, and Cambodian varieties of agarwood, we found that butyl hexadecanoate, butyl octadecanoate, bis(2-ethylhexyl) 1,2-benzenedicarboxylate, and squalene were common in the aforementioned four varieties of agarwoods. 2-(2-Phenylethyl) chromone derivatives were identified only in the incense smoke produced by Kynam agarwood, and were the major ingredient $(27.23 \%)$ in the same. In conclusion, this is the first study that analyzes chemical profiles of incense smoke produced by agarwood using HS GC-MS. Our data showed that 2-(2-phenylethyl) chromone derivatives could be used to assess quality of agarwoods. Moreover, HS GC/MS may be a useful tool for grading quality of agarwood.
\end{abstract}

Keywords: agarwood; headspace preheated system; gas chromatography-mass spectrometry; 2-(2-phenylethyl)chromone

\section{Introduction}

Agarwood, also called Gaharu, Chén-xīang, Jin-koh, Kyara, or Oud, is the resinous wood formed in the heartwood of Aquilaria trees. Aquilaria species, including A. sinensis (Lour.) Glig., A. agallocha Roxb., and A. malaccensis Lam., taxonomically belong to Thymelaeaceae and are found mainly in Southeast Asia, such as Vietnam, Indonesia, Laos, Cambodia, and Malaysia [1,2]. Agarwood and its oil are important and useful natural substances that have been used to produce valuable products. Agarwood has been used as incense in religious ceremonies for centuries as well as in traditional Chinese medicine for its sedative, aphrodisiac, carminative, and anti-emetic effects [3-9]. The value of agarwood depends on its quality. However, grading of agarwood tends to be discretionary and 
subjective, because agarwood is usually graded based on its physical properties, such as color, density, resin formation, by sensory panels [1,2,10]. Kynam, also known as Kanankoh in Japan, is regarded to have the highest quality among different varieties of agarwood [11]. The high resin content, better water sinking quality, and darker color of Kynam enable it to produce a special and pleasant scent [1]. Kynam has been considered to be the noblest spiritual wood, both in ancient societies and in modern times, because of its scarcity. At present, the cost of Kynam agarwood is about 350 Euro per gram [12]. Kynam agarwood has a very complex scent, and even grading professionals cannot easily distinguish it from others. Therefore, it is important and essential to develop analytic methods for judgment and grading of agarwood.

Chemical constituents of agarwood have been studied by several research teams [1,3,13]. Previous studies identified chemical constituents of agarwood essential oils or organic solvent extracts with column chromatography or spectroscopic techniques [14,15]. Other studies prepared agarwood essential oils, hydrodistillates, or solvent extracts for gas chromatography (GC) or multidimensional GC analysis [16,17]. However, analysis of chemical constituents in agarwood solvent extracts is not suitable for differentiation of agarwood because agarwood is usually used to produce incense smoke. A few research studies analyzed chemical ingredients in the incense smoke produced by heated agarwood [11,18,19]. For example, Ishihara et al. [11] analyzed chemical constituents in the incense smoke that was trapped in the Tenax TA adsorbent resin, and extracted in diethyl ether for GC and GC-mass spectrometry (MS) analysis. They found 53 chemical compounds in the incense smoke produced by high quality (Kanankoh) and low quality (Jinkoh) varieties of Vietnamese agarwood, and most of them were sesquiterpenes and aromatics. Hung et al. [18] prepared an agarwood extract by heating a sealed vial that contained agarwood powder in a water bath at $90{ }^{\circ} \mathrm{C}$ for $30 \mathrm{~min}$. The extract was absorbed in a solid-phase microextraction (SPME) needle with polydimethylsiloxane (PDMS) fiber for $30 \mathrm{~min}$, and then desorbed for GC-MS analysis. They found six peaks that could be used for evaluating the quality or price of the most expensive agarwood. Zhou et al. [19] collected incense smoke produced by agarwood and incense sticks, absorbed the different kinds of collected incense smoke in glass fiber pads, and extracted substances from the glass fiber pads with dichloromethane for GC-MS analysis. Nevertheless, these studies analyzed chemical constituents in the incense smoke that was either absorbed in matrices or extracted in solvents.

Analysis of chemical constituents in the incense smoke produced by agarwood may be an approach to grading of agarwood. Most of past research studies determine chemical ingredients in agarwood solvent extracts [13-17]. However, analysis of chemical constituents in solvent extracts is not suitable in the case of agarwood because it is usually used to produce incense smoke. In this work, we used a headspace (HS) preheating system that is combined with GC-MS (HS GC-MS) to extract heavier sample matrices for GC analysis. Incense smoke produced by heated agarwood was directly analyzed with GC-MS. We could obtain more authentic chemical profiles of incense smoke produced by agarwood without solid-phase extraction. Moreover, by comparing chemical constituents in the incense smoke produced by different grades of agarwood, we found that HS GC/MS-MS could be used to grade quality of agarwood.

\section{Results and Discussion}

\subsection{Morphological Observation of Various Grades of Agarwoods}

Agarwood is traditionally graded based on its physical properties, such as resin content, water sinking quality, color, scent/aroma, as well as agarwood-inducing method, formation time, and place of origin [1,2]. Both Kynam and Vietnamese varieties of agarwood showed first-grade colors and had softer scents (Figure 1A,B). However, the Kynam variety fully sank in water, while the Vietnam variety merely partially submerged in water. Both the Lao and Cambodian varieties of agarwood fully floated up, showed second-grade colors, and had sweet scents (Figure 1C,D). However, the sweet scent of Cambodian agarwood was induced by insect infection. 


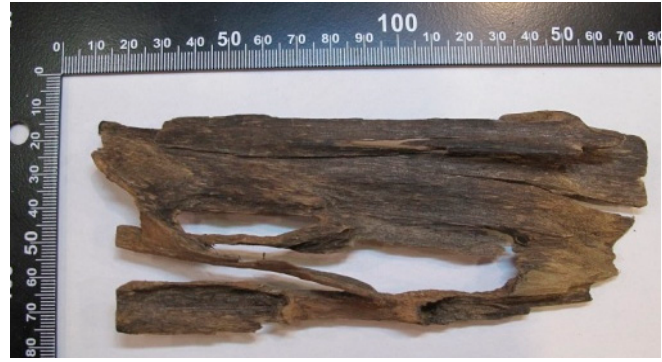

(A)

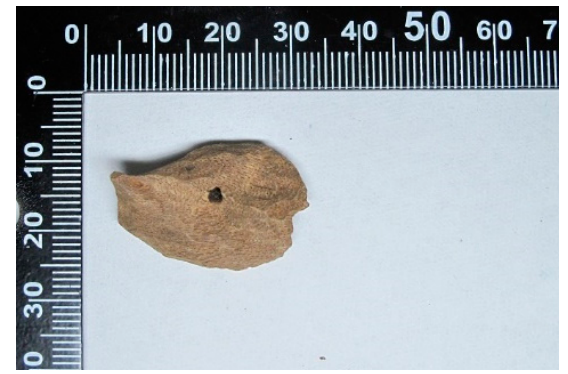

(C)

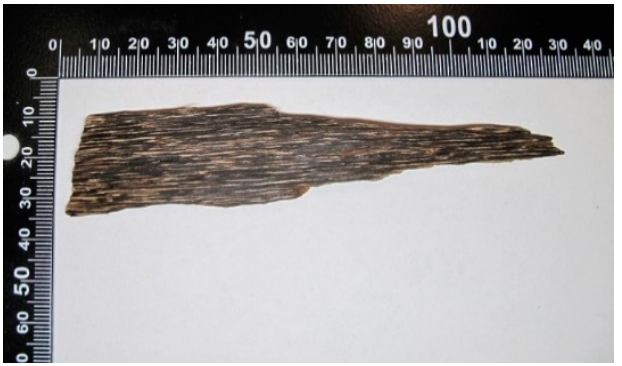

(B)

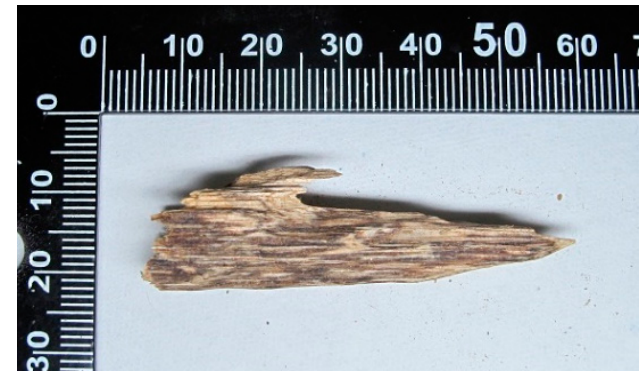

(D)

Figure 1. Morphological observation of Kynam (A), Vietnam (B), Liao (C), and Cambodian agarwoods (D).

\subsection{HS GC-MS/MS Analysis}

Chemical constituents of agarwood have been intensively studied by several research teams $[1,3,13]$. Different extraction methods have been developed to determine chemical composition of essential oils and related compounds from agarwood chips using GC, GC-MS, solid phase microextraction, GC-flame ionization detector, GC-olfactometry, or comprehensive two-dimensional gas chromatography $(\mathrm{GC} \times \mathrm{GC})[20]$. Most studies suggest that hydrodistillation is the method of choice for determining the essential oil content of agarwood [7]. Agarwood has only a slight scent at room temperature, and releases a pleasant aroma when heated. Burning incense is a common traditional practice in many families and most temples in Asia, for religious reasons and for its pleasant smell [1,2]. Incense smoke contains many kinds of fragrant sesquiterpenes and aromatics [11]. Without interphase mass transfer, how to obtain a large amount of incense smoke is an important parameter for efficient analysis. Therefore, we prepared the incense smoke of agarwood using a HS preheating system, and tested the incubation times and temperatures to obtain the maximum amount of analytes. Previous studies extracted agarwood oils through incubation at $40{ }^{\circ} \mathrm{C}$ for $10 \mathrm{~min}$, or at $90{ }^{\circ} \mathrm{C}$ for $30 \mathrm{~min}$ [18]. In this study, we prepared the incense smoke of agarwood through incubation at $150{ }^{\circ} \mathrm{C}$ for $30 \mathrm{~min}$, and injected the smoke into GC-MS/MS for chemical profiling analysis (Figure 2). 


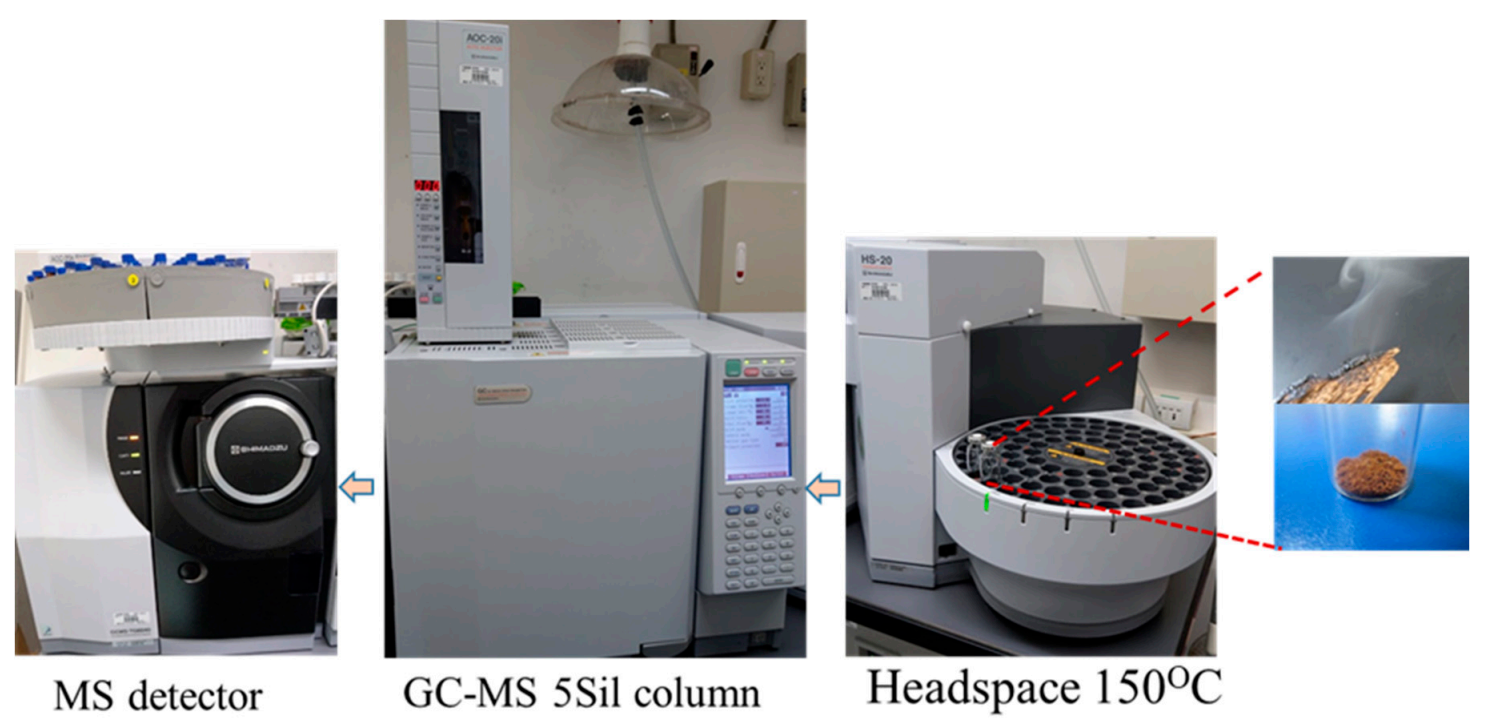

Figure 2. Experimental design and incense smoke detection process. Diagram shows that the incense smoke of agarwoods was generated by headspace preheated system and the smoke was injected directly to GC-MS for analysis.

\subsection{Chemical Profiles of Kynam Agarwoods Using HS GC/MS-MS}

Figure 3 and Figure S1 shows the GC chemical fingerprint of incense smoke produced by Kynam agarwood. The detection period was setup from $2.5 \mathrm{~min}$ to $65 \mathrm{~min}$. Forty peaks were identified in the GC-MS/MS analysis, and the identified compounds are summarized in Table 1. As shown in Figure 4, these compounds belong to different kinds of chemical categories, including aromatics $(33 \%)$, sesquiterpenes $(38 \%)$, alkanes $(8 \%)$, terpene $(3 \%)$, triterpenes $(3 \%)$, and others $(18 \%)$. Since sesquiterpenes and triterpenes were mostly identified in agarwoods, other terpenes, except sesquiterpenes and triterpenes, were classified as "terpene" in this study. Moreover, the major component in the incense smoke of Kynam was 2-(2-phenylethyl) chromone (27.23\%).

In 1993, Ishihara et al. [11] analyzed two kinds of agarwood incense smoke obtained with solvent extraction. Fifty components were identified in higher quality agarwood, while 36 components were identified in low quality agarwood. The incense smoke of both higher and low-quality agarwood was composed mainly of sesquiterpenes (53\% and $61 \%$, respectively) and aromatics ( $44 \%$ and $31 \%$, respectively). The major components in higher quality agarwood were guaia-1(10), 11-dien-15-oic acid (9.92\%), and 2-(2-phenylethyl) chromone (5.83\%). Hung et al. [18] identified 17 components that could be used as markers for classification and differentiation of agarwood. 2-(2-Phenylethyl) chromone derivatives (flindersiachromone) were also identified in most expensive agarwoods.

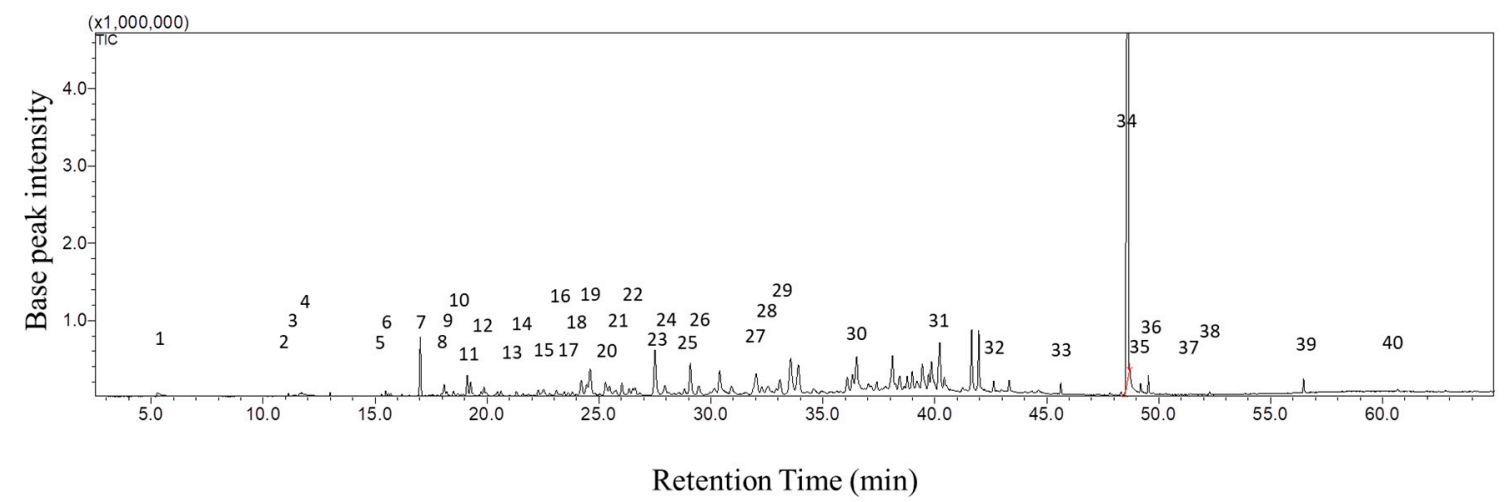

Figure 3. GC-MS chemical fingerprint of incense smoke from Kynam agarwood. Number indicates the identified compound, and the details of compounds are listed in Table 1. 
Table 1. List of compounds identified in the incense smoke from Kynam agarwood by GC-MS/MS.

\begin{tabular}{|c|c|c|c|c|c|c|c|}
\hline No. & Compound & LRI $^{a}$ & $\begin{array}{l}\text { Retention } \\
\text { Time (min) }\end{array}$ & $\begin{array}{c}\text { Content } \\
(\%)\end{array}$ & $\begin{array}{l}\text { Detected } \\
\mathrm{m} / \mathrm{z}\end{array}$ & $\begin{array}{l}\text { Chemical } \\
\text { Formula }\end{array}$ & Type $^{c}$ \\
\hline 1 & Benzaldehyde & 1581 & 5.296 & $0.17 \pm 0.05$ & 106 & $\mathrm{C}_{7} \mathrm{H}_{6} \mathrm{O}$ & $\mathrm{Ar}$ \\
\hline 2 & $\begin{array}{l}\text { exo-7-(2-Propenyl)bicyclo } \\
\text { [4.2.0]oct-1(2)-ene }\end{array}$ & 1720 & 11.137 & $0.04 \pm 0.02$ & 148 & $\mathrm{C}_{11} \mathrm{H}_{16}$ & Other \\
\hline 3 & 4-Methoxybenzaldehyde & 1731 & 11.617 & $0.14 \pm 0.05$ & 136 & $\mathrm{C}_{8} \mathrm{H}_{8} \mathrm{O}_{2}$ & $\mathrm{Ar}$ \\
\hline 4 & $\begin{array}{l}\text { 1,8-Nonadiene-3-yne, } \\
\text { 2,8-dimethyl-7-methylene- }\end{array}$ & 1734 & 11.717 & $0.04 \pm 0.03$ & 160 & $\mathrm{C}_{12} \mathrm{H}_{16}$ & Other \\
\hline 5 & Cubenene & 1818 & 15.273 & $0.03 \pm 0.02$ & 204 & $\mathrm{C}_{15} \mathrm{H}_{24}$ & $S$ \\
\hline 6 & $\alpha$-Longipinene & 1823 & 15.471 & $0.26 \pm 0.12$ & 204 & $\mathrm{C}_{15} \mathrm{H}_{24}$ & S \\
\hline 7 & $\begin{array}{c}\text { Tricyclo[5 } \\
\text { 2.2.0(1,6)]undecan-3-ol, } \\
\text { 2-methylene-6,8,8-trimethyl- }\end{array}$ & 1860 & 17.017 & $1.30 \pm 0.42$ & 220 & $\mathrm{C}_{15} \mathrm{H}_{24} \mathrm{O}$ & $\mathrm{Ar}$ \\
\hline 8 & Naphthalene & 1885 & 18.086 & $0.18 \pm 0.22$ & 202 & $\mathrm{C}_{15} \mathrm{H}_{22}$ & $\mathrm{Ar}$ \\
\hline 9 & $\delta$-Guaiene & 1888 & 18.196 & $0.13 \pm 0.08$ & 204 & $\mathrm{C}_{15} \mathrm{H}_{24}$ & S \\
\hline 10 & Nootkatene & 1895 & 18.502 & $0.12 \pm 0.06$ & 202 & $\mathrm{C}_{15} \mathrm{H}_{22}$ & $\mathrm{Ar}$ \\
\hline 11 & Kessane & 1910 & 19.119 & $0.39 \pm 0.29$ & 222 & $\mathrm{C}_{15} \mathrm{H}_{26} \mathrm{O}$ & $\mathrm{Ar}$ \\
\hline 12 & $\begin{array}{c}\text { 4a,5-Dimethyl-3-(prop-1-en-2 } \\
\text {-yl)-1,2,3,4,4a,5,6,7- } \\
\text { octahydronaphthalen-1-ol }\end{array}$ & 1928 & 19.863 & $0.31 \pm 0.07$ & 220 & $\mathrm{C}_{15} \mathrm{H}_{24} \mathrm{O}$ & $\mathrm{Ar}$ \\
\hline 13 & cis- $\alpha$-Santalol & 1962 & 21.312 & $0.28 \pm 0.09$ & 220 & $\mathrm{C}_{15} \mathrm{H}_{24} \mathrm{O}$ & S \\
\hline 14 & $\beta$-Vetivenene & 1969 & 21.593 & $0.10 \pm 0.02$ & 202 & $\mathrm{C}_{15} \mathrm{H}_{22}$ & $\mathrm{~T}$ \\
\hline 15 & $\gamma$-Eudesmol & 1998 & 22.799 & $0.06 \pm 0.06$ & 222 & $\mathrm{C}_{15} \mathrm{H}_{26} \mathrm{O}$ & $\mathrm{S}$ \\
\hline 16 & 10s,11s-Himachala-3(12),4-diene & 2017 & 23.61 & $0.27 \pm 0.12$ & 204 & $\mathrm{C}_{15} \mathrm{H}_{24}$ & Ar \\
\hline 17 & Agarospirol & 2022 & 23.808 & $0.21 \pm 0.04$ & 222 & $\mathrm{C}_{15} \mathrm{H}_{26} \mathrm{O}$ & $\mathrm{S}$ \\
\hline 18 & $\beta$-Guaiene & 2031 & 24.209 & $0.53 \pm 0.24$ & 204 & $\mathrm{C}_{15} \mathrm{H}_{24}$ & $\mathrm{~S}$ \\
\hline 19 & Cubenol & 2041 & 24.606 & $1.24 \pm 0.43$ & 222 & $\mathrm{C}_{15} \mathrm{H}_{26} \mathrm{O}$ & $\mathrm{S}$ \\
\hline 20 & $\alpha$-epi-7-epi-5-Eudesmol & 2057 & 25.296 & $0.95 \pm 0.28$ & 222 & $\mathrm{C}_{15} \mathrm{H}_{26} \mathrm{O}$ & $\mathrm{S}$ \\
\hline 21 & Bulnesol & 2074 & 26.021 & $0.26 \pm 0.18$ & 222 & $\mathrm{C}_{15} \mathrm{H}_{26} \mathrm{O}$ & S \\
\hline 22 & $\alpha$-Tetralone & 2089 & 26.624 & $0.53 \pm 0.26$ & 218 & $\mathrm{C}_{15} \mathrm{H}_{22} \mathrm{O}$ & $\mathrm{Ar}$ \\
\hline 23 & $\alpha$-Kessyl acetate & 2110 & 27.497 & $0.91 \pm 1.31$ & 280 & $\mathrm{C}_{17} \mathrm{H}_{28} \mathrm{O}_{3}$ & $\mathrm{Ar}$ \\
\hline 24 & $\begin{array}{c}(1 R, 7 S, E)-7-I s o p r o p y l-4,10- \\
\text { dimethylenecyclodec-5-enol }\end{array}$ & 2120 & 27.941 & $0.33 \pm 0.32$ & 220 & $\mathrm{C}_{15} \mathrm{H}_{24} \mathrm{O}$ & $\mathrm{Ar}$ \\
\hline 25 & 10-epi-Elemol & 2141 & 28.822 & $0.19 \pm 0.15$ & 222 & $\mathrm{C}_{15} \mathrm{H}_{26} \mathrm{O}$ & $\mathrm{S}$ \\
\hline 26 & Ledol & 2156 & 29.445 & $0.53 \pm 0.10$ & 222 & $\mathrm{C}_{15} \mathrm{H}_{26} \mathrm{O}$ & $\mathrm{S}$ \\
\hline 27 & Longiverbenone & 2217 & 32.017 & $0.82 \pm 0.74$ & 218 & $\mathrm{C}_{15} \mathrm{H}_{22} \mathrm{O}$ & Other \\
\hline 28 & Cryptomeridiol & 2230 & 32.552 & $1.95 \pm 1.10$ & 240 & $\mathrm{C}_{15} \mathrm{H}_{28} \mathrm{O}_{2}$ & S \\
\hline 29 & $\begin{array}{l}\text { 2aS,3aR,5aS,9bR)-2a,5a,9- } \\
\text { Trimethyl-2a,4,5,5a,6,7,8,9b- } \\
\text { octahydro-2H-naphtho[1- } \\
\text { b]oxireno[2-c]furan }\end{array}$ & 2243 & 33.081 & $0.40 \pm 0.45$ & 234 & $\mathrm{C}_{15} \mathrm{H}_{22} \mathrm{O}_{2}$ & $\mathrm{Ar}$ \\
\hline 30 & 10-epi- $\gamma$-Eudesmol & 2324 & 36.506 & $2.21 \pm 0.19$ & 222 & $\mathrm{C}_{15} \mathrm{H}_{26} \mathrm{O}$ & $\mathrm{S}$ \\
\hline 31 & Sandaracopimarinal & 2413 & 40.221 & $2.19 \pm 0.57$ & 286 & $\mathrm{C}_{20} \mathrm{H}_{30} \mathrm{O}$ & $\mathrm{Ar}$ \\
\hline 32 & $\gamma$-Gurjunene & 2470 & 42.629 & $0.52 \pm 0.10$ & 204 & $\mathrm{C}_{15} \mathrm{H}_{24}$ & $\mathrm{~S}$ \\
\hline 33 & Butyl hexadecanoate & 2542 & 45.628 & $0.75 \pm 0.46$ & 312 & $\mathrm{C}_{20} \mathrm{H}_{40} \mathrm{O}_{2}$ & Other \\
\hline
\end{tabular}


Table 1. Cont

\begin{tabular}{cccccccc}
\hline No. & Compound & LRI & $\begin{array}{c}\text { Retention } \\
\text { Time }(\mathbf{m i n})\end{array}$ & $\begin{array}{c}\text { Content } \\
\mathbf{( \% )}\end{array}$ & $\begin{array}{c}\text { Detected } \\
\boldsymbol{m} / \boldsymbol{z}\end{array}$ & $\begin{array}{c}\text { Chemical } \\
\text { Formula }\end{array}$ & Type $^{\mathbf{c}}$ \\
\hline 34 & 2-(2-Phenylethyl)chromone & 2613 & 48.64 & $26.19 \pm 1.0$ & 250 & $\mathrm{C}_{17} \mathrm{H}_{14} \mathrm{O}_{2}$ & Other \\
\hline 35 & Butyl octadecanoate & 2635 & 49.538 & $1.13 \pm 0.69$ & 340 & $\mathrm{C}_{22} \mathrm{H}_{44} \mathrm{O}_{2}$ & Other \\
\hline 36 & 1-Iodo-octacosane & 2640 & 49.743 & $0.05 \pm 0.03$ & 520 & $\mathrm{C}_{28} \mathrm{H}_{61} \mathrm{I}$ & $\mathrm{Ak}$ \\
\hline 37 & 1-Iodo-eicosane & 2680 & 51.447 & $0.09 \pm 0.06$ & 408 & $\mathrm{C}_{20} \mathrm{H}_{41} \mathrm{I}$ & $\mathrm{Ak}$ \\
\hline 38 & $\begin{array}{c}\text { Bis(2-ethylhexyl) } \\
\text { 1,2-benzenedicarboxylate }\end{array}$ & 2700 & 52.277 & $0.34 \pm 0.38$ & 390 & $\mathrm{C}_{24} \mathrm{H}_{38} \mathrm{O}_{4}$ & Other \\
\hline 39 & Squalene & 2806 & 56.472 & $1.19 \pm 1.30$ & 410 & $\mathrm{C}_{30} \mathrm{H}_{50}$ & Tri \\
\hline 40 & Nonacosane & 2900 & 60.669 & $0.08 \pm 0.05$ & 408 & $\mathrm{C}_{29} \mathrm{H}_{60}$ & $\mathrm{Ak}$ \\
\hline
\end{tabular}

${ }^{\text {a }}$ LRI: Linear retention index as tested on SH-Rxi-5Sil column using the series of C8-40 $n$-alkanes; ${ }^{\mathrm{b}}$ Values are the mean \pm standard error $(n=3) ;{ }^{c}$ Ak: alkane; Ar: aromatics; S: sesquiterpene; T: terpene; Tri: triterpene.

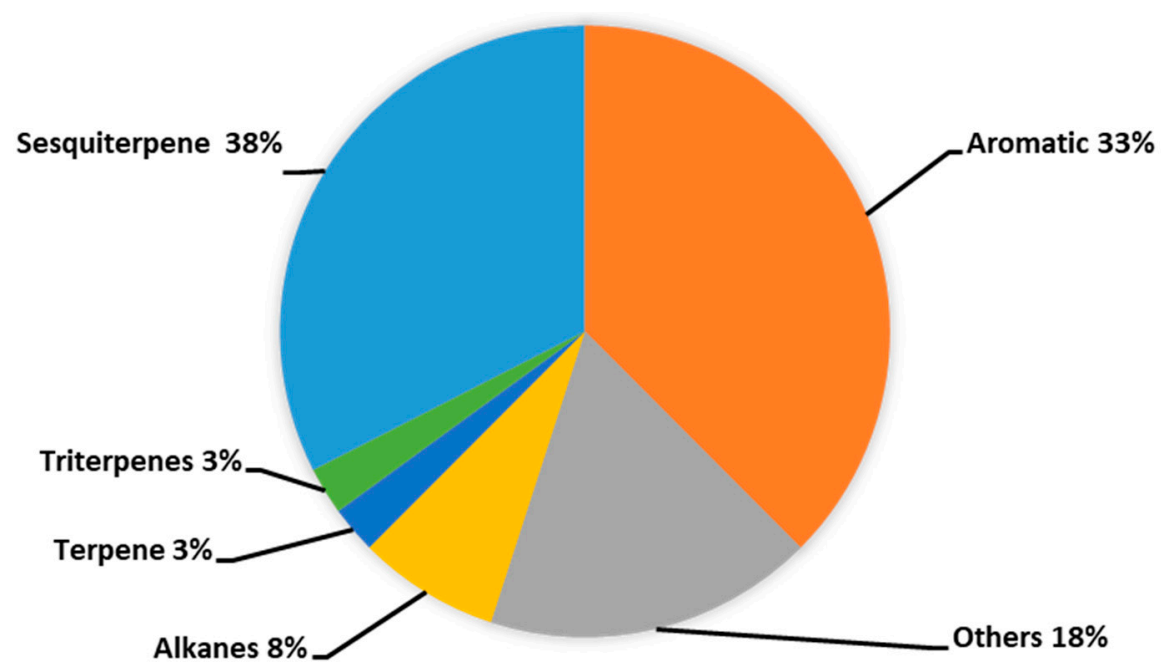

Figure 4. Pie chart showing chemical classification of 40 compounds identified in the incense smoke from Kynam agarwood.

A total of 132 constituents have been identified in solvent extracts from different varieties of agarwood in the past 5 decades, and they belong to sesquiterpenes (52\%), 2-(2-phenylethyl) chromone derivatives (41\%), and aromatics (1\%) [3]. In this study, we found that aromatics and sesquiterpenes were the two major chemical categories in the incense smoke of Kynam agarwood as analyzed with HS GC-MS. The difference may result from the extraction procedure or varieties of agarwood.

Sesquiterpenes and 2-(2-phenylethyl) chromone derivatives were the two predominant constituents in agarwood [2]. As chromone derivatives could not be pyrolyzed [11], Espinoza et al. [20] used direct analysis with real time time-of-flight mass spectrometry (DART-TOFMS) to analyze 60 commercial agarwood chips without extraction, and identified the presence of key ions and the characteristics of 2-(2-phenylethyl) chromones. They found that 8-16 target chromone ions were present in each sample. These results showed that the highly oxidized agarwood chromones were specific to Aquilaria spp.

\subsection{Chemical Profiles of Different Grades of Agarwoods Using HS GC/MS-MS}

To further compare chemical constituents in the incense smoke produced by Vietnamese, Lao, and Cambodian varieties of agarwood with those in the same produced by the Kynam variety, we prepared the incense smoke using a HS preheating system, and the smoke was directly injected to GC for chemical identification. Lao agarwood shares similar physical properties with Cambodian 
agarwood, and both varieties exhibited similar patterns in GC (Figure 5 and Figure S2). A total of 110 constituents were identified in the incense smoke produced by four varieties of agarwood, including 40 compounds in the Kynam variety, 25 compounds in the Vietnamese variety, 45 compounds in the Lao variety, and 31 compounds in the Cambodian variety. Four compounds were commonly identified in the four varieties of agarwood, while 28, 13, 26, and 19 compounds were specifically identified in the Kynam, Vietnamese, Lao, and Cambodian varieties of agarwood, respectively (Figure 5D and Table 2). Butyl hexadecanoate, butyl octadecanoate, bis(2-ethylhexyl) 1,2-benzenedicarboxylate, and aqualene were commonly found in the incense smoke produced by the four varieties of agarwood. Ishihara et al. [11] analyzed the smoke profiles of two different varieties of Vietnamese agarwood that were absorbed in Tenax TA, and found small amounts of benzaldehyde and 2-(2-phenylethyl) chromone were present in both varieties of Kynam (Kanakoh) agarwood. Ismail et al. [21] analyzed the chemical constituents of agarwood oil that was absorbed in PDMS and divinylbenzene-carboxen-PDMS, and found that 4-phenyl-2-butanone was one of the major compounds that contributed to the scent. 4-Methoxy-benzaldehyde was the first compound identified in the incense smoke of agarwood in this study, whereas it had not been identified in agarwood smoke or oils in other studies. These findings indicate that the identification of constituents in agarwood is affected by adsorbents. Therefore, more authentic chemical profiles of agarwood can be achieved with the use of incense smoke without matrix absorption.

Table 2. Compounds commonly or specifically present in the incense smoke of agarwoods.

\begin{tabular}{|c|c|c|c|c|c|}
\hline Compound & $\begin{array}{l}\text { Retention } \\
\text { Time (min) }\end{array}$ & LRI & $\begin{array}{l}\text { Detected } \\
\qquad m / z\end{array}$ & $\begin{array}{l}\text { Chemical } \\
\text { Formula }\end{array}$ & Agarwoods $^{a}$ \\
\hline Butyl hexadecanoate & 45.628 & 2542 & 312 & $\mathrm{C}_{17} \mathrm{H}_{40} \mathrm{O}_{2}$ & $\mathrm{~K}, \mathrm{~V}, \mathrm{~L}, \mathrm{C}$ \\
\hline Butyl octadecanoate & 49.538 & 2635 & 340 & $\mathrm{C}_{22} \mathrm{H}_{44} \mathrm{O}_{2}$ & $\mathrm{~K}, \mathrm{~V}, \mathrm{~L}, \mathrm{C}$ \\
\hline Bis(2-ethylhexyl) 1,2-benzenedicarboxylate & 52.277 & 2700 & 390 & $\mathrm{C}_{24} \mathrm{H}_{38} \mathrm{O}_{4}$ & $\mathrm{~K}, \mathrm{~V}, \mathrm{~L}, \mathrm{C}$ \\
\hline Squalene & 56.472 & 2800 & 410 & $\mathrm{C}_{30} \mathrm{H}_{50}$ & $\mathrm{~K}, \mathrm{~V}, \mathrm{~L}, \mathrm{C}$ \\
\hline Benzaldehyde & 5.296 & 1581 & 106 & $\mathrm{C}_{7} \mathrm{H}_{6} \mathrm{O}$ & K \\
\hline 4-methoxy-Benzaldehyde & 11.617 & 1731 & 136 & $\mathrm{C}_{8} \mathrm{H}_{8} \mathrm{O}_{2}$ & $\mathrm{~K}$ \\
\hline $\begin{array}{l}\text { 1,8-Nonadien-3-yne, } \\
\text { 2,8-dimethyl-7-methylene- }\end{array}$ & 11.717 & 1734 & 160 & $\mathrm{C}_{12} \mathrm{H}_{16}$ & $\mathrm{~K}$ \\
\hline Cubenene & 15.275 & 1818 & 204 & $\mathrm{C}_{15} \mathrm{H}_{24}$ & K \\
\hline$\alpha$-Longipinene & 15.471 & 1823 & 204 & $\mathrm{C}_{15} \mathrm{H}_{24}$ & K \\
\hline $\begin{array}{l}\text { Tricyclo[5.2.2.0(1,6)]undecan-3-ol, } \\
\text { 2-methylene-6,8,8-trimethyl- }\end{array}$ & 17.017 & 1860 & 220 & $\mathrm{C}_{15} \mathrm{H}_{24} \mathrm{O}$ & $\mathrm{K}$ \\
\hline Naphthalene & 18.086 & 1885 & 202 & $\mathrm{C}_{15} \mathrm{H}_{22}$ & $\mathrm{~K}$ \\
\hline$\delta$-Guaiene & 18.196 & 1888 & 204 & $\mathrm{C}_{15} \mathrm{H}_{24}$ & $\mathrm{~K}$ \\
\hline Nootkatene & 18.502 & 1895 & 202 & $\mathrm{C}_{15} \mathrm{H}_{22}$ & $\mathrm{~K}$ \\
\hline $\begin{array}{l}\text { 4a,5-Dimethyl-3-(prop-1-en-2-yl)- } \\
\text { 1,2,3,4,4a,5,6,7-octahydronaphthalen-1-ol }\end{array}$ & 19.863 & 1928 & 220 & $\mathrm{C}_{15} \mathrm{H}_{24} \mathrm{O}$ & $\mathrm{K}$ \\
\hline cis- $\alpha$-Santalol & 21.312 & 1962 & 220 & $\mathrm{C}_{15} \mathrm{H}_{24} \mathrm{O}$ & K \\
\hline$\beta$-Vetivenene & 21.593 & 1969 & 202 & $\mathrm{C}_{15} \mathrm{H}_{22}$ & K \\
\hline$\gamma$-Eudesmol & 22.799 & 1998 & 222 & $\mathrm{C}_{15} \mathrm{H}_{26} \mathrm{O}$ & $\mathrm{K}$ \\
\hline 10s,11s-Himachala-3(12),4-diene & 23.610 & 2017 & 204 & $\mathrm{C}_{15} \mathrm{H}_{24}$ & $\mathrm{~K}$ \\
\hline$\beta$-Guaiene & 24.209 & 2031 & 204 & $\mathrm{C}_{15} \mathrm{H}_{24}$ & K \\
\hline Cubenol & 24.606 & 2041 & 222 & $\mathrm{C}_{15} \mathrm{H}_{26} \mathrm{O}$ & K \\
\hline$\alpha$-Tetralone & 26.624 & 2089 & 218 & $\mathrm{C}_{15} \mathrm{H}_{22} \mathrm{O}$ & $\mathrm{K}$ \\
\hline$\alpha$-Kessyl acetate & 27.497 & 2110 & 280 & $\mathrm{C}_{17} \mathrm{H}_{28} \mathrm{O}_{3}$ & K \\
\hline
\end{tabular}


Table 2. Cont.

\begin{tabular}{|c|c|c|c|c|c|}
\hline Compound & $\begin{array}{l}\text { Retention } \\
\text { Time (min) }\end{array}$ & LRI & $\begin{array}{l}\text { Detected } \\
\qquad m / z\end{array}$ & $\begin{array}{l}\text { Chemical } \\
\text { Formula }\end{array}$ & Agarwoods $^{a}$ \\
\hline $\begin{array}{l}(1 R, 7 S, E)-7-\text { Isopropyl-4,10- } \\
\text { dimethylenecyclodec-5-enol }\end{array}$ & 27.941 & 2120 & 220 & $\mathrm{C}_{15} \mathrm{H}_{24} \mathrm{O}$ & K \\
\hline Ledol & 29.445 & 2156 & 222 & $\mathrm{C}_{15} \mathrm{H}_{26} \mathrm{O}$ & K \\
\hline Longiverbenone & 32.017 & 2217 & 218 & $\mathrm{C}_{15} \mathrm{H}_{22} \mathrm{O}$ & K \\
\hline $\begin{array}{l}\text { 2aS,3aR,5aS,9bR)-2a,5a,9-Trimethyl- } \\
\text { 2a,4,5,5a,6,7,8,9b-octahydro- } 2 H- \\
\text { naphtho[1-b]oxireno[2-c]furan }\end{array}$ & 33.081 & 2243 & 234 & $\mathrm{C}_{15} \mathrm{H}_{22} \mathrm{O}_{2}$ & $\mathrm{~K}$ \\
\hline 10-epi- $\gamma$-Eudesmol & 36.506 & 2324 & 222 & $\mathrm{C}_{15} \mathrm{H}_{26} \mathrm{O}$ & $\mathrm{K}$ \\
\hline Sandaracopimarinal & 40.221 & 2413 & 286 & $\mathrm{C}_{20} \mathrm{H}_{30} \mathrm{O}$ & K \\
\hline$\gamma$-Gurjunene & 42.629 & 2470 & 204 & $\mathrm{C}_{15} \mathrm{H}_{24}$ & $\mathrm{~K}$ \\
\hline 2-(2-Phenylethyl)chromone & 48.640 & 2613 & 250 & $\mathrm{C}_{17} \mathrm{H}_{14} \mathrm{O}_{2}$ & $\mathrm{~K}$ \\
\hline 1-iodo-Triacontane & 49.743 & 2640 & 548 & $\mathrm{C}_{22} \mathrm{H}_{44} \mathrm{O}_{2}$ & $\mathrm{~K}$ \\
\hline Eicosane, 1-iodo- & 51.447 & 2680 & 408 & $\mathrm{C}_{20} \mathrm{H}_{41} \mathrm{I}$ & K \\
\hline $\begin{array}{l}\text { 2-Isopropenyl-4a,8-dimethyl-1,2,3,4,4a,5,6,7- } \\
\text { octahydronaphthalene }\end{array}$ & 17.354 & 1868 & 204 & $\mathrm{C}_{15} \mathrm{H}_{24}$ & $\mathrm{~L}$ \\
\hline Dehydrodeoxybaimuxinol & 19.844 & 1927 & 220 & $\mathrm{C}_{15} \mathrm{H}_{24} \mathrm{O}$ & $\mathrm{L}$ \\
\hline Rosifoliol & 22.267 & 1985 & 222 & $\mathrm{C}_{15} \mathrm{H}_{26} \mathrm{O}$ & $\mathrm{L}$ \\
\hline $\begin{array}{c}\text { 1H-Cyclopropa[a]naphthalene, } \\
\text { 1a,2,3,3a,4,5,6,7b-octahydro-1,1,3a,7- } \\
\text { tetramethyl-, [1aR-(1a } \alpha, 3 \mathrm{a} \alpha, 7 \mathrm{~b} \alpha)]-\end{array}$ & 23.598 & 2017 & 204 & $\mathrm{C}_{15} \mathrm{H}_{24}$ & $\mathrm{~L}$ \\
\hline Guaiol acetate & 23.692 & 2019 & 222 & $\mathrm{C}_{15} \mathrm{H}_{26} \mathrm{O}$ & $\mathrm{L}$ \\
\hline Isovalencenol & 24.782 & 2045 & 218 & $\mathrm{C}_{15} \mathrm{H}_{22} \mathrm{O}$ & $\mathrm{L}$ \\
\hline $\begin{array}{l}\text { 4-isopropenyl-1-methoxymethoxymethyl- } \\
\text { cyclohexene }\end{array}$ & 25.046 & 2051 & 196 & $\mathrm{C}_{12} \mathrm{H}_{20} \mathrm{O}_{2}$ & $\mathrm{~L}$ \\
\hline trans- $\alpha$-Bisabolene & 25.447 & 2061 & 204 & $\mathrm{C}_{15} \mathrm{H}_{24}$ & $\mathrm{~L}$ \\
\hline$\beta$-Patchoulene & 25.667 & 2066 & 204 & $\mathrm{C}_{15} \mathrm{H}_{24}$ & $\mathrm{~L}$ \\
\hline Androstan-17-one, 3-ethyl-3-hydroxy-, $(5 \alpha)$ - & 25.938 & 2072 & 318 & $\mathrm{C}_{21} \mathrm{H}_{34} \mathrm{O}_{2}$ & $\mathrm{~L}$ \\
\hline $\begin{array}{c}\text { Acetate, }(2,4 a, 5,8 a-t e t r a m e t h y l- \\
\text { 1,2,3,4,4a,7,8,8a-octahydro-1- } \\
\text { naphthalenyl) ester }\end{array}$ & 26.337 & 2082 & 250 & $\mathrm{C}_{16} \mathrm{H}_{26} \mathrm{O}_{3}$ & $\mathrm{~L}$ \\
\hline Dehydrofukinone & 27.707 & 2115 & 218 & $\mathrm{C}_{15} \mathrm{H}_{22} \mathrm{O}$ & $\mathrm{L}$ \\
\hline Neoisolongifolene, 8,9-epoxy- & 33.235 & 2246 & 218 & $\mathrm{C}_{15} \mathrm{H}_{22} \mathrm{O}$ & $\mathrm{L}$ \\
\hline $\begin{array}{l}\text { 6-Isopropenyl-4,8a-dimethyl-1,2,3,5,6,7,8,8a- } \\
\text { octahydro-naphthalen-2-ol }\end{array}$ & 34.576 & 2278 & 220 & $\mathrm{C}_{15} \mathrm{H}_{24} \mathrm{O}$ & $\mathrm{L}$ \\
\hline (S)-cis-Verbenol & 36.717 & 2329 & 152 & $\mathrm{C}_{10} \mathrm{H}_{16} \mathrm{O}$ & $\mathrm{L}$ \\
\hline $\begin{array}{c}\text { Card-20(22)-enolide, } \\
\text { 3,5,14,19-tetrahydroxy-, }(3 \beta, 5 \beta) \text { - }\end{array}$ & 38.348 & 2368 & 406 & $\mathrm{C}_{23} \mathrm{H}_{34} \mathrm{O}_{6}$ & $\mathrm{~L}$ \\
\hline Espatulenol & 39.248 & 2390 & 220 & $\mathrm{C}_{15} \mathrm{H}_{24} \mathrm{O}$ & $\mathrm{L}$ \\
\hline $\begin{array}{l}\text { 5-Isopropenyl-2-methylcyclopent-1- } \\
\text { enecarboxaldehyde }\end{array}$ & 39.504 & 2396 & 150 & $\mathrm{C}_{10} \mathrm{H}_{14} \mathrm{O}$ & $\mathrm{L}$ \\
\hline Levoverbenone & 39.650 & 2399 & 150 & $\mathrm{C}_{10} \mathrm{H}_{14} \mathrm{O}$ & $\mathrm{L}$ \\
\hline $\begin{array}{c}\text { Cyclobutene, } \\
\text { 4,4-dimethyl-1-(2,7-octadienyl)- }\end{array}$ & 39.908 & 2405 & 190 & $\mathrm{C}_{14} \mathrm{H}_{22}$ & $\mathrm{~L}$ \\
\hline Alloaromadendrene oxide-(1) & 40.407 & 2417 & 220 & $\mathrm{C}_{15} \mathrm{H}_{24} \mathrm{O}$ & $\mathrm{L}$ \\
\hline
\end{tabular}


Table 2. Cont.

\begin{tabular}{|c|c|c|c|c|c|}
\hline Compound & $\begin{array}{l}\text { Retention } \\
\text { Time (min) }\end{array}$ & LRI & $\begin{array}{l}\text { Detected } \\
\qquad m / z\end{array}$ & $\begin{array}{l}\text { Chemical } \\
\text { Formula }\end{array}$ & Agarwoods $^{a}$ \\
\hline $\begin{array}{l}\text { 3-Oxatricyclo[20.8.0.0(7,16)] triaconta- } \\
\text { 1(22),7(16),9,13,23,29-hexaene }\end{array}$ & 41.858 & 2452 & 406 & $\mathrm{C}_{29} \mathrm{H}_{42} \mathrm{O}$ & $\mathrm{L}$ \\
\hline Andrographolide & 42.100 & 2458 & 350 & $\mathrm{C}_{20} \mathrm{H}_{30} \mathrm{O}_{5}$ & $\mathrm{~L}$ \\
\hline $\begin{array}{l}\text { Docosa-2,6,10,14,18-pentaen-22-al, } \\
\text { 2,6,10,15,18-pentamethyl-, all-trans }\end{array}$ & 48.062 & 2600 & 384 & $\mathrm{C}_{27} \mathrm{H}_{44} \mathrm{O}$ & $\mathrm{L}$ \\
\hline 2,6,10-trimethyl-Tetradecane & 49.731 & 2639 & 240 & $\mathrm{C}_{17} \mathrm{H}_{36}$ & $\mathrm{~L}$ \\
\hline Heptadecanal & 51.965 & 2693 & 254 & $\mathrm{C}_{17} \mathrm{H}_{34} \mathrm{O}$ & $\mathrm{L}$ \\
\hline 4,5-di-epi-Aristolochene & 17.343 & 1868 & 204 & $\mathrm{C}_{15} \mathrm{H}_{24}$ & $\mathrm{~V}$ \\
\hline$\alpha$-Costal & 25.156 & 2054 & 218 & $\mathrm{C}_{15} \mathrm{H}_{22} \mathrm{O}$ & $\mathrm{V}$ \\
\hline $\begin{array}{l}\text { 6,7-Dimethyl-1,2,3,5,8,8a- } \\
\text { hexahydronaphthalene }\end{array}$ & 25.964 & 2073 & 162 & $\mathrm{C}_{12} \mathrm{H}_{18}$ & V \\
\hline Dihydrocolumellarin & 39.222 & 2389 & 234 & $\mathrm{C}_{15} \mathrm{H}_{22} \mathrm{O}_{2}$ & $\mathrm{~V}$ \\
\hline Columellarin & 40.694 & 2424 & 232 & $\mathrm{C}_{15} \mathrm{H}_{20} \mathrm{O}_{2}$ & $\mathrm{~V}$ \\
\hline Isovelleral & 44.081 & 2505 & 232 & $\mathrm{C}_{15} \mathrm{H}_{20} \mathrm{O}_{2}$ & $\mathrm{~V}$ \\
\hline Octadecanoic acid & 45.149 & 2530 & 284 & $\mathrm{C}_{18} \mathrm{H}_{36} \mathrm{O}_{2}$ & $\mathrm{~V}$ \\
\hline 1-Hexacosene & 45.755 & 2545 & 364 & $\mathrm{C}_{26} \mathrm{H}_{52}$ & $\mathrm{~V}$ \\
\hline Pentadecanal- & 46.405 & 2560 & 226 & $\mathrm{C}_{15} \mathrm{H}_{30} \mathrm{O}$ & $\mathrm{V}$ \\
\hline$n$-Pentadecanol & 47.783 & 2593 & 228 & $\mathrm{C}_{15} \mathrm{H}_{32} \mathrm{O}$ & $\mathrm{V}$ \\
\hline 2-Nonadecanone & 48.071 & 2600 & 282 & $\mathrm{C}_{19} \mathrm{H}_{38} \mathrm{O}$ & $\mathrm{V}$ \\
\hline Dotriacontane, 1-iodo- & 52.442 & 2704 & 576 & $\mathrm{C}_{32} \mathrm{H}_{65} \mathrm{I}$ & $\mathrm{V}$ \\
\hline Tetracosanal & 53.568 & 2731 & 352 & $\mathrm{C}_{24} \mathrm{H}_{48} \mathrm{O}$ & V \\
\hline$\alpha$-Ylangene & 14.047 & 1789 & 204 & $\mathrm{C}_{15} \mathrm{H}_{24}$ & $\mathrm{C}$ \\
\hline 5,5-dimethyl-4-(3-oxobutyl)-Spiro[2.5]octane & 15.489 & 1824 & 208 & $\mathrm{C}_{14} \mathrm{H}_{24} \mathrm{O}$ & $\mathrm{C}$ \\
\hline Diepicedrene-1-oxide & 17.013 & 1860 & 220 & $\mathrm{C}_{15} \mathrm{H}_{24} \mathrm{O}$ & $\mathrm{C}$ \\
\hline 7-epi- $\alpha$-Cadinene & 17.220 & 1865 & 204 & $\mathrm{C}_{15} \mathrm{H}_{24}$ & C \\
\hline$\alpha$-Guaiene & 17.385 & 1869 & 204 & $\mathrm{C}_{15} \mathrm{H}_{24}$ & C \\
\hline Alloaromadendrene oxide-(2) & 19.874 & 1928 & 220 & $\mathrm{C}_{15} \mathrm{H}_{24} \mathrm{O}$ & $\mathrm{C}$ \\
\hline Diethyl phthalate & 21.888 & 1976 & 222 & $\mathrm{C}_{12} \mathrm{H}_{14} \mathrm{O}_{4}$ & C \\
\hline Hinesol & 23.982 & 2026 & 222 & $\mathrm{C}_{15} \mathrm{H}_{26} \mathrm{O}$ & $\mathrm{C}$ \\
\hline cis-Eudesm-6-en-11-ol & 24.456 & 2037 & 222 & $\mathrm{C}_{15} \mathrm{H}_{26} \mathrm{O}$ & $\mathrm{C}$ \\
\hline Longipinane, $(E)-$ & 25.371 & 2059 & 206 & $\mathrm{C}_{15} \mathrm{H}_{26}$ & C \\
\hline Pentadecanoic acid & 35.990 & 2312 & 242 & $\mathrm{C}_{15} \mathrm{H}_{30} \mathrm{O}_{2}$ & C \\
\hline Palmitoleic acid & 39.168 & 2388 & 254 & $\mathrm{C}_{16} \mathrm{H}_{30} \mathrm{O}_{2}$ & $\mathrm{C}$ \\
\hline Hexadecane & 45.448 & 2537 & 226 & $\mathrm{C}_{16} \mathrm{H}_{34}$ & $\mathrm{C}$ \\
\hline trans-Geranylgeraniol & 48.077 & 2600 & 290 & $\mathrm{C}_{20} \mathrm{H}_{34} \mathrm{O}$ & C \\
\hline Tetradecanal & 48.432 & 2608 & 212 & $\mathrm{C}_{14} \mathrm{H}_{28} \mathrm{O}$ & $\mathrm{C}$ \\
\hline Hexadecanal & 50.261 & 2652 & 240 & $\mathrm{C}_{16} \mathrm{H}_{32} \mathrm{O}$ & $\mathrm{C}$ \\
\hline Octacosane & 51.439 & 2680 & 394 & $\mathrm{C}_{28} \mathrm{H}_{58}$ & $\mathrm{C}$ \\
\hline Oxirane, hexadecyl- & 51.970 & 2693 & 268 & $\mathrm{C}_{18} \mathrm{H}_{36} \mathrm{O}$ & C \\
\hline Heptacosane & 53.037 & 2718 & 380 & $\mathrm{C}_{27} \mathrm{H}_{56}$ & $\mathrm{C}$ \\
\hline
\end{tabular}

\footnotetext{
${ }^{\text {a }}$ K: Kynam; V: Vietnam; L: Liao; C: Cambodian.
} 


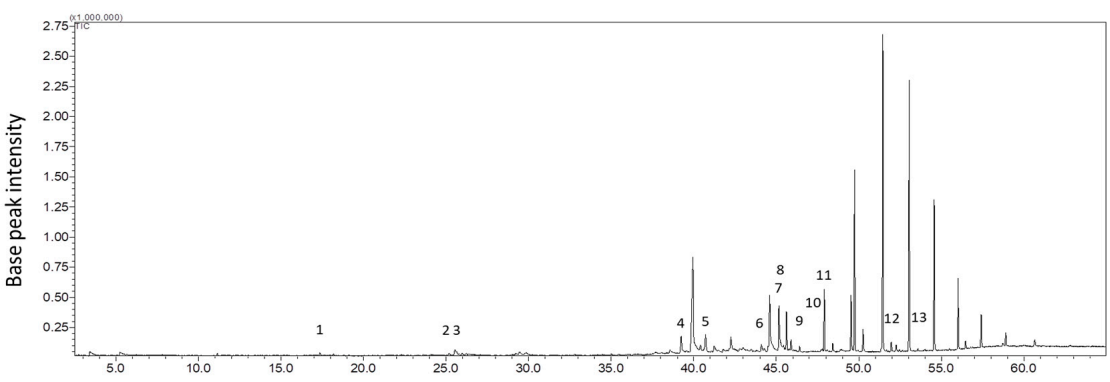

(A)

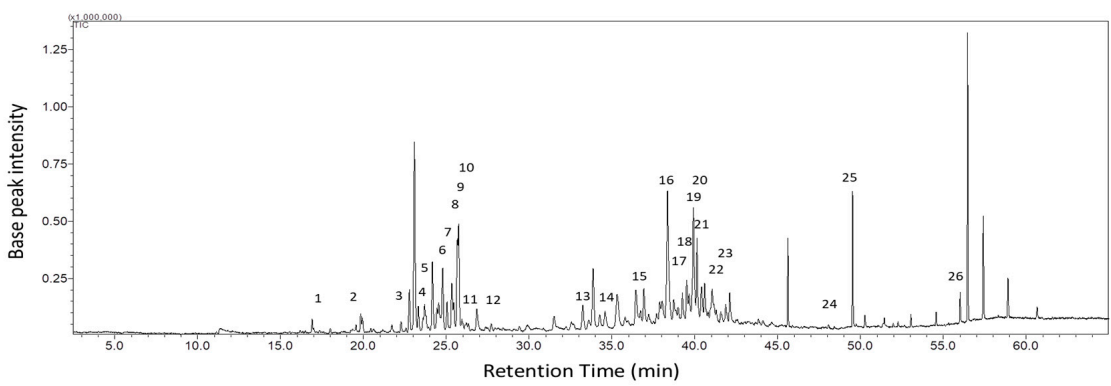

(B)

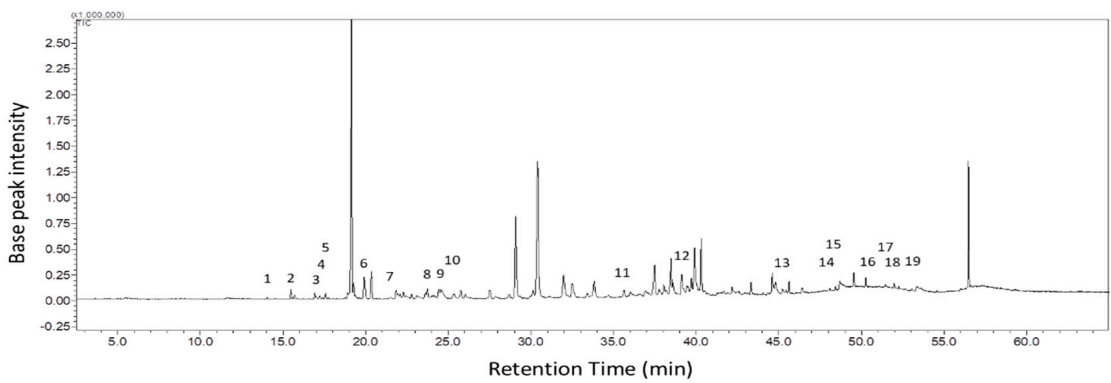

(C)

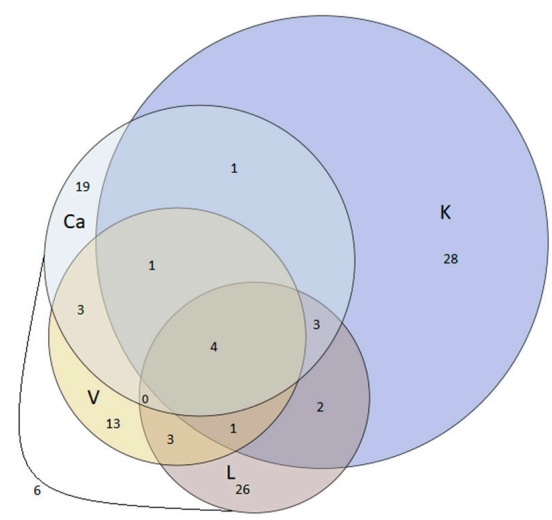

(D)

Figure 5. GC-MS chemical fingerprint of incense smoke from agarwoods. (A) Vietnam agarwood. (B) Liao agarwood. (C) Cambodian agarwood. Number indicates the compound specifically present in the agarwood. (D) Venn diagram of 110 compounds identified in the incense smoke from Kynam (K), Vietnam (V), Liao (L), and Cambodian (Ca) agarwoods. 


\subsection{Chemical Ingredients of Different Grades of Agarwoods}

Sesquiterpenoids and 2-(2-phenylethyl) chromone derivatives are two predominant constituents in agarwood [1-3]. Sesquiterpenoids, the essential ingredients in luxury perfumes, have been identified in different varieties of agarwood. In addition to sesquiterpenes, 2-(2-phenylethyl) chromone derivative (flindersiachromone, 2-phenethyl-4H-chromen-4-one) is another key reference constituent in agarwood. 2-(2-Phenylethyl)chromone derivatives can only be extracted in solvents or supercritical $\mathrm{CO}_{2}$, and are never found in hydrodistillates [22-24]. Substitute chromones in different grades of agarwood have also been studied. 2-(2-Phenylethyl) chromone derivatives can be detected in $76 \%$ of agarwood chips using direct analysis with DART-TOFMS without chemical extraction; however, DART-TOFMS is only used for distinguishing Aquilaria genus from others instead of agarwood grading [20]. One previous study showed that 2-(2-phenylethyl)chromone derivatives were found in incense smoke produced by Vietnamese agarwood, with a GC peak area of $5.83 \%$ in Kanankoh (high quality variety of agarwood) [11]. Another study also showed that 2-(2-phenylethyl)chromone derivatives were present only in expensive agarwood [15]. In this study, we found that 2-(2-phenylethyl) chromones were identified in the incense smoke of the Kynam variety $(27.23 \%)$, but not in the same of Vietnamese, Lao, or Cambodian variety. So far, 39 different 2-(2-phenylethyl) chromone derivatives have been identified in different varieties of agarwood [20,25]. In line with these studies, our findings suggest that analysis of 2-(2-phenylethyl) chromone derivatives can be used to assess quality of agarwood. Moreover, the contents of 2-(2-phenylethyl) chromone derivative in triple duplicates of Kynam smoke were $25.18 \%, 27.23 \%$, and $26.17 \%$ (Supplementary Materials Figure S1). Therefore, our data suggested that the HS GC/MS developed in this study can be a useful tool for grading quality of agarwood through direct analysis of incense smoke.

\section{Materials and Methods}

\subsection{Reagents}

All chemicals were purchased from Sigma (St. Louis, MO, USA), unless indicated.

\subsection{Agarwood Materials}

All natural agarwoods, including Kynam, Vietnam, Liao and Cambodia, were purchased from Chenglin Inc. (Yunlin, Taiwan). Agarwoods used in this study were identified and qualified by The International Tropical Timber Organization. The voucher specimen has been deposited in Development Center for Biotechnology. The agarwood chips were pulverized into a fine powder before use.

\subsection{Sample Preparation}

The incense smoke of agarwood was prepared using HS preheated system. Agarwood powder $(50 \mathrm{mg}$ ) was weighted and placed in a $20 \mathrm{~mL}$ glass vial. The vial was sealed with Teflon, shaken, and incubated at $150{ }^{\circ} \mathrm{C}$ for $30 \mathrm{~min}$ in a headspace autosampler oven (Model HS-20, Shimadzu Corporation, Kyoto, Japan).

\subsection{GC-MS/MS Analysis}

Chemical constituents of incense smoke were analyzed by GS-MS/MS. Incense smoke (1 mL) was injected into GC-MS/MS (Model GC-2010 Plus and GCMS-TQ8040, Shimadzu Corporation, Kyoto, Japan). Temperature of sample line and transfer line was kept at $150{ }^{\circ} \mathrm{C}$ Ultra-high purity helium $(99.99 \%)$ was used as the carrier gas at a flow rate of $5.1 \mathrm{~mL} / \mathrm{min}$. The chromatographic separation was conducted with SH-Rxi-5Sil MS capillary column (30 $\mathrm{m} \times 0.25 \mathrm{~mm}$ inner diameter, $0.25 \mu \mathrm{m}$ thickness, Shimadzu Corporation, Kyoto, Japan). The column temperature program was set as follows: $60^{\circ} \mathrm{C}$ initial temperature and subsequently ramped to $140{ }^{\circ} \mathrm{C}$ at a rate of $6{ }^{\circ} \mathrm{C} / \mathrm{min}$, held at $140{ }^{\circ} \mathrm{C}$ for $5 \mathrm{~min}$, ramped to $160^{\circ} \mathrm{C}$ at a rate of $2{ }^{\circ} \mathrm{C} / \mathrm{min}$, held at $160^{\circ} \mathrm{C}$ for $5 \mathrm{~min}$, ramped to $280^{\circ} \mathrm{C}$ at a rate 
of $5{ }^{\circ} \mathrm{C} / \mathrm{min}$, held at $280^{\circ} \mathrm{C}$ for $10 \mathrm{~min}$. The GC-MS interface temperature was maintained at $250{ }^{\circ} \mathrm{C}$. Electron ionization was used as the ionization method, and the ion source temperature was set at $230{ }^{\circ} \mathrm{C}$. The mass spectra obtained with full scan and the mass ranged from $m / z 45$ to 550 were used for determining the unknown components.

Raw data were processed using GCMS solution Version 4.41 (Shimadzu Corporation, Kyoto, Japan). Mass spectral fragmentation patterns were compared with those stored in the NIST Mass Spectral Library (nist14), which is built up by using the pure substances and the mass spectra from literatures. In order to get the linear retention index values of the volatile compounds, a series of $n$-alkanes calibration standard (C8-C40) was run in the same condition (Figure S3). A Venn diagram was generated by eulerr program of $R$ package [26].

\section{Conclusions}

So far, grading of agarwoods is flexible and subjective, because of the absence of standard grading system. Scent is the most important parameter for the quality assessment of agarwoods. In this work, we established a simple, automated, robust, and high-throughput method to analyze the incense smoke generated from a minimum amount of agarwood sample by HS GC-MS/MS. In comparison with the incense smoke constituents in Vietnam, Liao, and Cambodian agarwoods, we found that Kynam agarwood was composed of more complex constituents. Moreover, 2-(2-phenylethyl)chromone derivative was present in Kynam instead of Vietnam, Liao, and Cambodia agarwoods, suggesting that the content of 2-(2-phenylethyl)chromone derivative could be applied to grade the quality of agarwoods.

Supplementary Materials: The Supplementary Materials are available online.

Author Contributions: Methodology, W.-Y.K. and S.-C.H.; Supervision, T.-Y.H. and K.-T.L.; Writing—original draft, W.-Y.K. and C.-Y.H.; Writing-review \& editing, C.-Y.H., T.-Y.H. and L.-T.L.

Funding: This research was funded by Ministry of Economic Affairs (Small Business Innovation Research Program 1Z1030845), Ministry of Science and Technology (MOST105-2320-B-039-017-MY3 and MOST107-2320-B-039-029-MY3), and China Medical University (CMU104-H-01 and CMU104-H-02).

Conflicts of Interest: The authors declare no conflict of interest.

\section{References}

1. Kalra, R.; Kaushik, N. A review of chemistry, quality and analysis of infected agarwood tree (Aquilaria sp.). Phytochem. Rev. 2017, 16, 1045-1079. [CrossRef]

2. Liu, Y.; Wei, J.; Gao, Z.; Zhang, Z.; Lyu, J. A review of quality assessment and grading for agarwood. Chin. Herb. Med. 2017, 9, 22-30. [CrossRef]

3. Chen, H.Q.; Wei, J.H.; Yang, J.S.; Zhang, Z.; Yang, Y.; Gao, Z.H.; Sui, C.; Gong, B. Chemical constituents of agarwood originating from the endemic genus Aquilaria plants. Chem. Biodivers. 2012, 9, 236-250. [CrossRef] [PubMed]

4. Chen, H.; Yang, Y.; Xue, J.; Wei, J.; Zhang, Z.; Chen, H. Comparison of compositions and antimicrobial activities of essential oils from chemically stimulated agarwood, wild agarwood and healthy Aquilaria sinensis (Lour.) gilg trees. Molecules 2011, 16, 4884-4896. [CrossRef] [PubMed]

5. Hashim, Y.Z.; Kerr, P.G.; Abbas, P.; Mohd Salleh, H. Aquilaria spp. (agarwood) as source of health beneficial compounds: A review of traditional use, phytochemistry and pharmacology. J. Ethnopharmacol. 2016, 189, 331-360. [CrossRef] [PubMed]

6. Tay, P.Y.; Tan, C.P.; Abas, F.; Yim, H.S.; Ho, C.W. Assessment of extraction parameters on antioxidant capacity, polyphenol content, epigallocatechin gallate (EGCG), epicatechin gallate (ECG) and iriflophenone 3-C- $\beta$-glucoside of agarwood (Aquilaria crassna) young leaves. Molecules 2014, 19, 12304-12319. [CrossRef] [PubMed]

7. Wang, S.; Yu, Z.; Wang, C.; Wu, X.; Guo, P.; Wei, J. Chemical constituents and pharmacological activity of agarwood and Aquilaria plants. Molecules 2018, 23, E342. [CrossRef] [PubMed] 
8. Wang, S.; Wang, C.; Peng, D.; Liu, X.; Wu, C.; Guo, P.; Wei, J. Agarwood essential oil displays sedative-hypnotic effects through the GABAergic system. Molecules 2017, 22, E2190. [CrossRef] [PubMed]

9. Wang, S.L.; Tsai, Y.C.; Fu, S.L.; Cheng, M.J.; Chung, M.I.; Chen, J.J. 2-(2-Phenylethyl)-4H-chromen-4-one derivatives from the resinous wood of Aquilaria sinensis with anti-inflammatory effects in LPS-induced macrophages. Molecules 2018, 23, E289. [CrossRef] [PubMed]

10. Ismail, S.N.; Maulidiani, M.; Akhtar, M.T.; Abas, F.; Ismail, I.S.; Khatib, A.; Ali, N.A.M.; Shaari, K. Discriminative analysis of different grades of gaharu (Aquilaria malaccensis Lamk.) via ${ }^{1} \mathrm{H}-\mathrm{NMR}-\mathrm{based}$ metabolomics using PLS-DA and random forests classification models. Molecules 2017, 22, E1612. [CrossRef] [PubMed]

11. Ishihara, M.; Tsuneya, T.; Uneyama, K. Components of the agarwood smoke on heating. J. Essent. Oil Res. 1993, 5, 419-423. [CrossRef]

12. The Scent of Kyara (Oud). Available online: https://www.onoda.ch/news/2018/1/20/kyara-agarwood (accessed on 26 September 2018).

13. Liao, G.; Dong, W.H.; Yang, J.L.; Li, W.; Wang, J.; Mei, W.L.; Dai, H.F. Monitoring the chemical profile in agarwood formation within one year and speculating on the biosynthesis of 2-(2-phenylethyl)chromones. Molecules 2018, 23, E1261. [CrossRef] [PubMed]

14. Ito, T.; Kakino, M.; Tazawa, S.; Watarai, T.; Oyama, M.; Maruyama, H.; Araki, Y.; Hara, H.; Iinuma, M. Quantification of polyphenols and pharmacological analysis of water and ethanol-based extracts of cultivated agarwood leaves. J. Nutr. Sci. Vitaminol. (Tokyo) 2012, 58, 136-142. [CrossRef] [PubMed]

15. Tajuddin, S.N.; Yusoff, M.M. Chemical composition of volatile oils of Aquilaria malaccensis (Thymelaeaceae) from Malaysia. Nat. Prod. Commun. 2010, 5, 1965-1968. [PubMed]

16. Gao, X.; Xie, M.; Liu, S.; Guo, X.; Chen, X.; Zhong, Z.; Wang, L.; Zhang, W. Chromatographic fingerprint analysis of metabolites in natural and artificial agarwood using gas chromatography-mass spectrometry combined with chemometric methods. J. Chromatogr. B 2014, 967, 264-273. [CrossRef] [PubMed]

17. Mei, W.L.; Yang, D.L.; Wang, H.; Yang, J.L.; Zeng, Y.B.; Guo, Z.K.; Dong, W.H.; Li, W.; Dai, H.F. Characterization and determination of 2-(2-phenylethyl)chromones in agarwood by GC-MS. Molecules 2013, 18, 12324-12345. [CrossRef] [PubMed]

18. Hung, C.H.; Lee, C.Y.; Yang, C.L.; Lee, M.R. Classification and differentiation of agarwoods by using non-targeted HS-SPME-GC/MS and multivariate analysis. Anal. Methods 2014, 6, 7449-7456. [CrossRef]

19. Zhou, R.; An, Q.; Pan, X.W.; Yang, B.; Hu, J.; Wang, Y.H. Higher cytotoxicity and genotoxicity of burning incense than cigarette. Environ. Chem. Lett. 2015, 13, 465-471. [CrossRef]

20. Espinoza, E.O.; Lancaster, C.A.; Kreitals, N.M.; Hata, M.; Cody, R.B.; Blanchette, R.A. Distinguishing wild from cultivated agarwood (Aquilaria spp.) using direct analysis in real time and time of-flight mass spectrometry. Rapid Commun. Mass Spect. 2014, 28, 281-289. [CrossRef] [PubMed]

21. Ahmaed, D.T.; Kulkarni, A.D. Sesquiterpenes and chromones of agarwood: A review. Malays. J. Chem. 2017, $19,33-58$.

22. Ismail, N.; Azah, M.A.N.; Jamil, M.; Rahiman, M.H.F.; Tajuddin, S.N.; Taib, M.N. Analysis of high quality agarwood oil chemical compounds by means of SPME/GC-MS and Z-SCORE technique. Malays. J. Anal. Sci. 2013, 17, 403-413.

23. Jong, P.L.; Tsan, P.; Mohamed, R. Gas chromatography-mass spectrometry analysis of agarwood extracts from mature and juvenile Aquilaria malaccensis. Inter. J. Agric. Biol. 2014, 16, 644-648.

24. Liu, Y.Y.; Chen, H.Q.; Yang, Y.; Zhang, Z.; Wei, J.H.; Meng, H. Whole-tree agarwood-inducing technique: An efficient novel technique for producing high-quality agarwood in cultivated Aquilaria sinensis trees. Molecules 2013, 18, 3086-3106. [CrossRef] [PubMed] 
25. Richter, J.; Schellenberg, I. Comparison of different extraction methods for the determination of essential oils and related compounds from aromatic plants and optimization of solid-phase microextraction/gas chromatography. Anal. Bioanal. Chem. 2007, 387, 2207-2217. [CrossRef] [PubMed]

26. R Core Team. R: A Language and Environment for Statistical Computing; R Foundation for Statistical Computing: Vienna, Austria, 2013.

Sample Availability: Samples of the agarwoods are available from the authors.

(C) 2018 by the authors. Licensee MDPI, Basel, Switzerland. This article is an open access article distributed under the terms and conditions of the Creative Commons Attribution (CC BY) license (http://creativecommons.org/licenses/by/4.0/). 